\title{
Tailoring the enhanced frequency shift in two-dimensional photonic clusters
}

\author{
A. A. Asatryan ${ }^{\mathrm{a}, *}$, L. C. Botten ${ }^{\mathrm{a}}$, N.A. Nicorovici, ${ }^{\mathrm{a}, \mathrm{b}}$ R. C. McPhedran ${ }^{\mathrm{b}}$, C.M. de Sterke ${ }^{\mathrm{b}}$ \\ ${ }^{a}$ Centre for Ultrahigh-bandwidth Devices for Optical Systems (CUDOS) and Department of Mathematical Sciences, \\ University of Technology, Sydney, PO Box 123, Broadway, NSW 2007, Australia \\ ${ }^{\mathrm{b}}$ CUDOS and School of Physics, University of Sydney, NSW 2006, Australia
}

\begin{abstract}
The frequency shift of sources embedded in a two-dimensional photonic cluster composed of circular cylinders is considered and the effects of cluster shape and orientation are investigated. It is found that by carefully tailoring the shape of the cluster the frequency shift can be greatly enhanced even for relatively small clusters-behaviour that can be understood in terms of the poles of the scattering matrix. It is also found that the frequency shift is a strong function of the orientation of the photonic cluster.
\end{abstract}

Key words: Lamb shift, multiple scattering, photonic crystals PACS: 42.25.Fx, 42.50.-p, 03.50.De

\section{Introduction}

The subject of photonic crystals $(\mathrm{PC})$ is one of the most active areas of research in optics [1]. Attention is being focussed on applications that exploit both band gap effects [2], opening up the possibility of miniaturised, integrated photonic circuits, and also their capacity to suppress or enhance spontaneous emission by modifying the local density of statesheralding the promise of new sources such as ultralow threshold lasers and high efficiency nano-scale LEDs embedded within PC devices. Indeed, the original two papers in the field of photonic crystals emphasised these key characteristics and their application $[3,4]$.

The ability of PCs to influence the density of states and affect the emission properties of atoms/sources leads naturally to the consideration

\footnotetext{
* Corresponding author.

Email address: ara.asatrian@uts.edu.au (A. A. Asatryan).
}

of quantum effects, including the control of the spontaneous emission rate [5], the non-Markovian character of radiative decay [6], and the localisation of super-radiance near the photonic band edge [7].

One of the fundamental radiation effects is the Lamb shift (or frequency shift) [8], the initial calculation of which considered an infinite photonic crystal [9]. However, as all realistic structures are finite, it is important to consider the influence of a finite scatterer on the frequency shift. In our earlier paper [10], we showed that the frequency shift is a strong function of both the size and the shape of the cluster. In this paper, we build on these results and show that it is possible to "tailor" the shape of the cluster to give rise to a strongly enhanced frequency shift. We also consider the effects of the cluster's orientation on the frequency shift.

Our treatment follows that of Morawits [11], in which the radiation reaction mechanism is employed - an approach that was successful in modeling experimental data in the 1970s [12]. In 
our approach, we consider a classical problem, noting that modes are essentially the same in each quantum or classical treatments. We consider a two-dimensional, square symmetric, photonic cluster composed of circular cylinders of infinite length in which the field is TM $\left(E_{\|}\right)$polarised, i.e., with the electric field aligned with the cylinder axes. In Fig.1, the black dot represents an embedded (line) source in the finite $2 \mathrm{D}$ cluster. In our simulations, the cylinders have a common radius of $a=0.3 d$, where $d$ is the separation between the neighbouring cylinders, and a common refractive index $n=3$ embedded in a material with the refractive index $n_{b}=1$. This structure has a total band gap in the wavelength range $2.989<\lambda / d<3.769$.

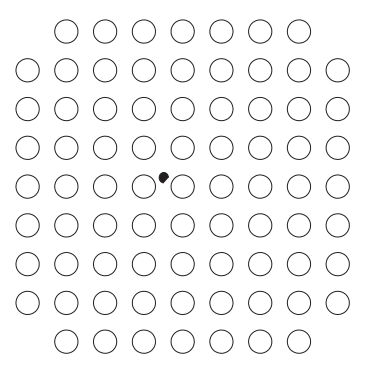

Fig. 1. The geometry of the problem. The black dot denotes the source.

In this framework the frequency shift is determined by the Green function of the problem [10] which can be conveniently calculated using the multipole method since the cylinders have circular cross section [13].

The Green function, can be expressed in the form

$$
G\left(\boldsymbol{r}, \boldsymbol{r}_{s}\right)=G_{0}\left(\boldsymbol{r}, \boldsymbol{r}_{s}\right)+G_{R}(\boldsymbol{r}),
$$

in which the first term $\left(G_{0}\left(\boldsymbol{r}, \boldsymbol{r}_{s}\right)=H_{0}^{(1)}(k n \mid \boldsymbol{r}-\right.$ $\left.\left.\boldsymbol{r}_{s} \mid\right) /(4 i)\right)$ is the "undressed" Green function, corresponding to an isolated source in a homogeneous material of refractive index $n$, while the second term in (1) is the scattered field $G_{R}(\boldsymbol{r})$. From this, we may compute the frequency shift (relative to the free space shift), given by the real part of the scattered component of the Green function at the source location $G_{R}\left(\boldsymbol{r}_{s}\right)$. That is,

$$
\delta \omega=-2 \gamma \operatorname{Re} G_{R}\left(\boldsymbol{r}_{s}\right),
$$

where $\gamma=P / W$ is the free space damping coefficient, given as a ratio of the radiated energy per second $P$ divided by the total energy $W[13]$. For further details on this effective and accurate approach, see Refs [10,13].

We now consider the application of the method and plot in Fig. 2 the frequency shift, as a function

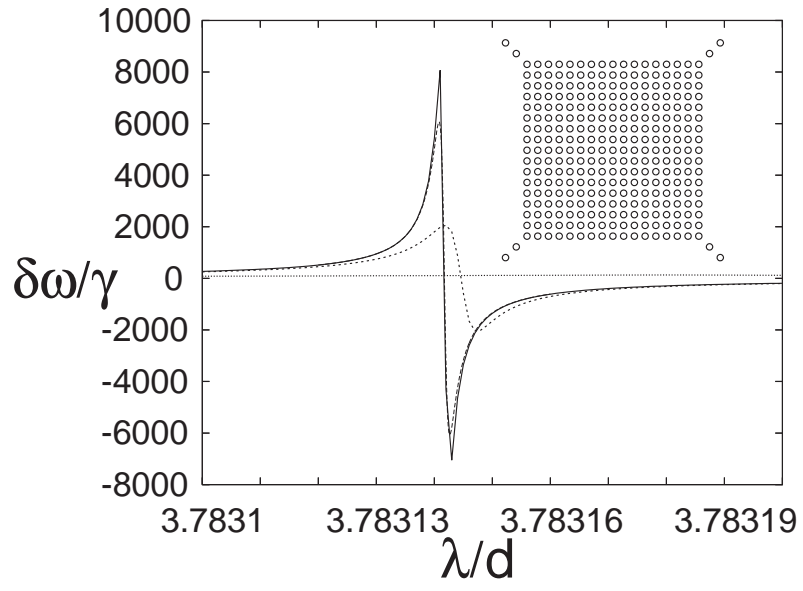

Fig. 2. Frequency shift vs wavelength for the cluster shown in the inset (solid line), for the cluster with one extra cylinder in each corner (fine line), for the corresponding square $(17 \times 17)$ cluster (dotted line), and for a cluster derived from the square cluster by removing the four corner cylinders (flat curve).

of the wavelength for a source located at the centre of the central cylinder in a $17 \times 17$ cluster of cylinders that form a square array (dotted line). The solid line of Fig. 2 is for the cluster depicted in the inset, while the frequency shift for a cluster derived from the square cluster by removing the four corner cylinders corresponds to visual accuracy to the central line. Clearly evident is a strongly enhanced frequency shift in the vicinity of the band gap edge at $\lambda / d=3.769$ for the corresponding infinite array. The frequency shift for the square cluster (dashed curve) reaches a value of $\delta \omega / \gamma=2058$ at $\lambda / d=$ 3.783142 , while the removal of the four corner cylinders reduces the maximum shift to only $\delta \omega / \gamma=120$ at $\lambda / d=3.78318$ (the flat curve in Fig. 2). However, the addition of four cylinders (one in each corner in a diagonal orientation) to the square cluster increases the maximum frequency shift to $\delta \omega / \gamma \approx$ 6000 at $\lambda / d=3.783140$, while the addition of a further four, diagonal corner cylinders further increases the frequency shift to $\delta \omega / \gamma \approx 8000$ at $\lambda / d=$ 3.783140. Note, that the adopted method is valid given $\delta \omega / \omega \ll 1$, otherwise a full quantum mechanical approach is needed.

The frequency shift is clearly a strong function of the cluster shape and, by careful tailoring, a large frequency shift is achievable even for the relatively small clusters. Indeed, the frequency shift achieved for the cluster of 297 cylinders shown in the inset of Fig. 2 is similar to that achieved with a larger $(25 \times$ 25) square cluster with 625 cylinders [13]. In this case, we note that the band gap is associated with 
the $\Gamma M$ propagation direction and so the additional or removal of cylinders along this direction strongly affects the confinement of the radiation, and hence the frequency shift.

Such behaviour can be understood in terms of the pole structure of the multipole scattering operator

$$
\boldsymbol{S}=(\boldsymbol{I}-\boldsymbol{R} \boldsymbol{H})^{-1} .
$$

In this, $\boldsymbol{R}$ is a diagonal, multipole reflection matrix that takes into account the material properties of the cylinders (i.e., radius and refractive index) while the $\boldsymbol{H}$ matrix accommodates the multiple scattering processes which are governed by the geometry of the structure. Since the structure is open (finite), there are radiation losses and so the poles of $\boldsymbol{S}$ must lie in the complex plane.

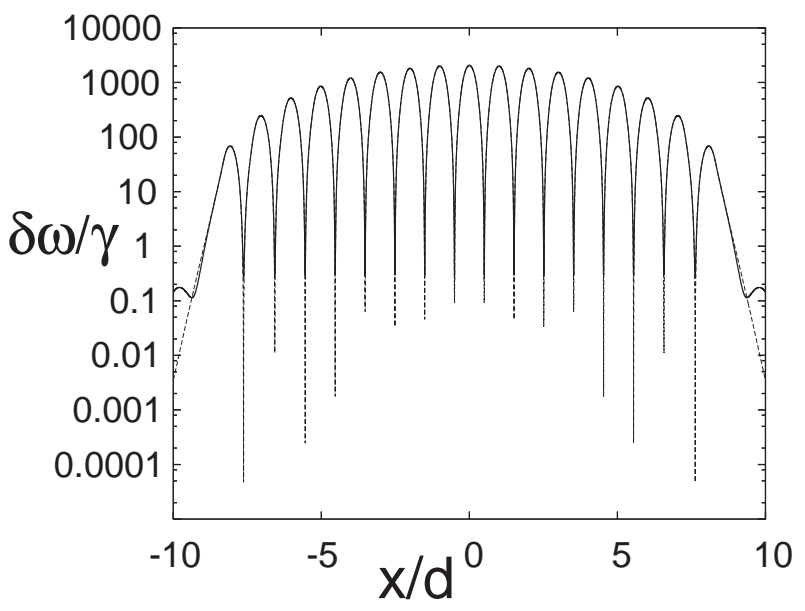

Fig. 3. Frequency shift as a function of the position $x / d$ (solid line); and the dashed line is the intensity of the mode profile along the $x$ axes.

In Fig. 3 (solid line) we plot the frequency shift of a sources for a square $17 \times 17$ cluster for $\lambda / d=$ 3.783142 (i.e., the wavelength at which the maximum frequency shift occurs) as a function of the position along the $x$ axis (i.e, a horizontal line through the middle of the cluster). Superimposed on this is the intensity (dotted line) of the leaky mode $|V|^{2}$ and it is clear that the frequency shift of the source is directly associated with this leaky mode which has a complex wavelength of $\lambda / d=3.78314461+$ $i 0.000002723$. This mode has a high quality factor of $Q=694664$ and so is confined within the cluster for a long time, allowing it to interact strongly with the source and thus shifting its frequency.

By examining more closely the structure of the mode, computed from the null space of $\boldsymbol{I}-\boldsymbol{R H}$ using the multipole method, we learn that the multi- pole coefficients of all orders $m \neq 0$ are small for all cylinders, and that only the monopole order is significant. Accordingly, there is no phase cancellation from the different cylinders, thus explaining the strong effect that is evident. (Note that although the higher multipole coefficients are small, the problem cannot be solved by retaining only the leading monopole coefficient; the higher order terms are necessary for a physically converged solution.) In the light of this, we can see that the enhancement of the frequency shift is the collective effect of all cylinders. The leaky mode can be viewed as a FabryPerot resonance associated with multiple reflections from the edges of the cluster. Our particular mode is associated with the symmetric "dielectric" mode of the corresponding infinite structure. Indeed, it is evident from Fig. 3 that the modal field is concentrated inside the cylinders. Note that in the case of an infinite structure, this mode is a standing mode at the edge of the gap [14].

This standing wave pattern can be seen from the distribution of the Poynting vector (see Fig. 4(b)) and, as expected, the main leakage of energy occurs from the corners of the cluster. The Fig. 4(a) plots the intensity $\left(|V|^{2}\right)$ distribution of the mode and it is evident that this peaks in the centre of the cylinders.

It is also interesting to consider the effects on the frequency shift of the orientation of the cluster. In Fig. 5(a) we plot the frequency shift as a function of wavelength for a square cluster, the rows of which are conventionally oriented along the $\Gamma X$ direction (see inset). Correspondingly, Fig. 5(b) displays the frequency shift as a function of wavelength for a cluster whose rows are oriented in the $\Gamma M$ direction. The solid vertical line in Fig. 5(a) indicates the location of the band gap for the corresponding infinite structure. Clearly evident in Fig. 5 is the very strong effect that cluster orientation has on the frequency shift. For the cluster with the $\Gamma X$ orientation, the frequency shift is very strong reaching a maximal value of $\delta \omega / \gamma=2058$. However, for the $\Gamma M$ orientation, the maximal frequency shift is only $\delta \omega / \gamma=12$, occurring for $\lambda / d=4.52$ at which the corresponding infinite structure has a partial band gap in the $\Gamma X$ direction. A weak enhancement of $\delta \omega / \gamma=4$ also occurs for the $\Gamma X$ oriented cluster at $\lambda / d=4.52$.

In conclusion, we have calculated the frequency (Lamb) shift of sources embedded in finite two dimensional photonic clusters and have determined computationally that the frequency shift is a strong function of the cluster shape. By carefully tailoring the shape of the cluster it is possible to achieve ex- 
(a)

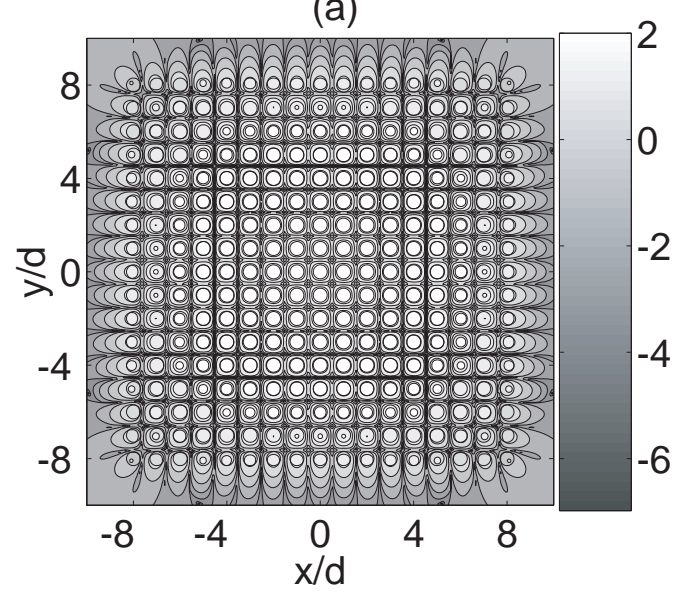

(b)

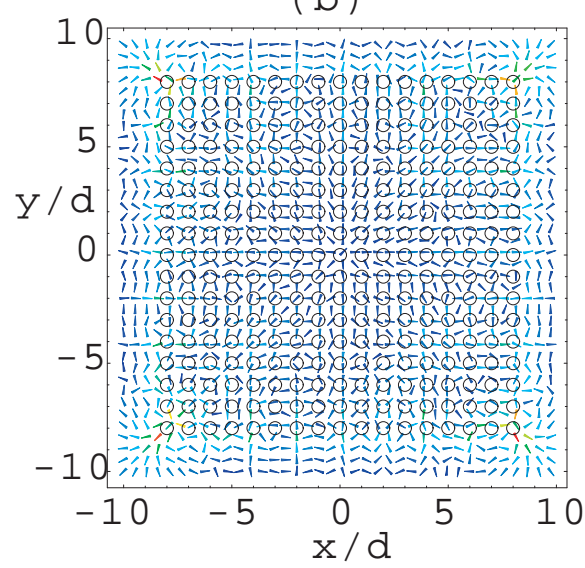

Fig. 4. Intensity distribution of the leaky mode as a function of position (a) with the logarithmic scale in the color bar; the Poynting vector as a function of the position (b).

tremely high frequency shifts, even for a cluster of only moderate size. As was demonstrated, this behaviour can be explained in terms of the leaky modes of the structure, which are associated with poles of the scattering operator. For wavelengths close to the band gap edge, these poles are close to the real axis of the complex wavelength plane and hence the associated leaky mode has a long lifetime, allowing it to interact with the source and thereby shift its frequency.

Support from the Australian Research Council under the its Centres of Excellence program is acknowledged.

\section{References}

[1] J.D Joannopoulos, R.D. Meade, and J. N. Winn Photonic crystals: Molding the flow of light, (Princeton
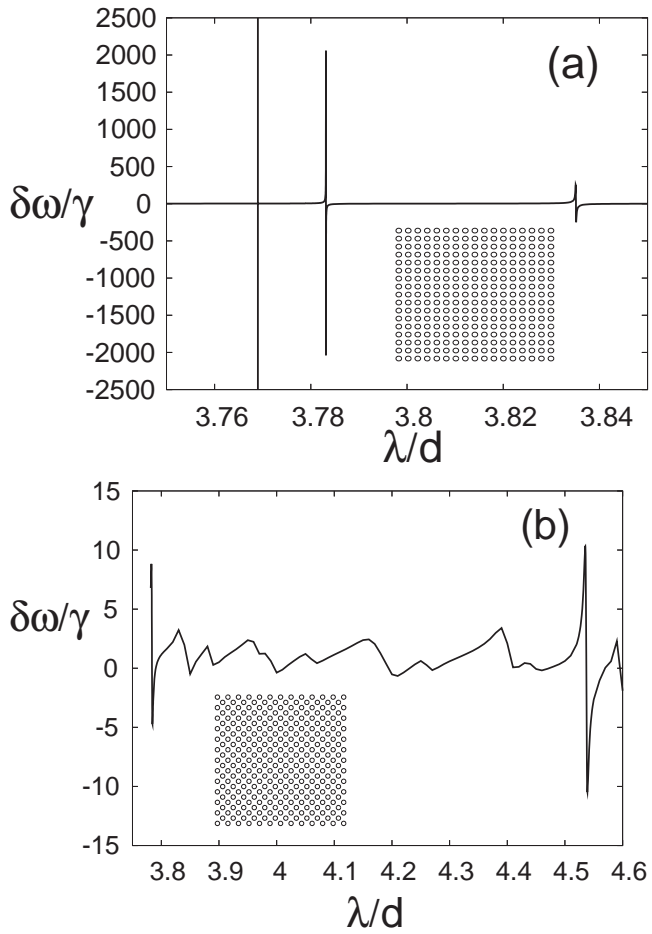

Fig. 5. The influence of orientation on the frequency shift; (a): for a cluster whose rows are oriented along the $\Gamma X$ direction; (b): for a cluster with rows oriented along the $\Gamma M$ direction.

University Press, New Jersey, 1995).

[2] Photonic crystals and light localization in the 21st century, (Proceedings of the NATO Advanced Study Institute on Photonic Crystals and Light Localization), Ed. C. M. Soukoulis, Limin Hersonissou, Crete, Greece, June 18-30, 2000.

[3] S. John, Phys. Rev. Lett. 58, 2486 (1987).

[4] I. Yablonowitz, Phys. Rev. Lett. 58, 2059 (1987).

[5] T. Quang, M. Woldeyohannes, S. John, and G. S. Agrawal, Phys. Rev. Lett., 79, 5238 (1997).

[6] K. Busch, N. Vats, S. John, and B. C. Sanders, Phys. Rev. E, 62, 4251 (2000).

[7] S. John, T. Quang, Phys. Rev. Lett., 74, 3419 (1995).

[8] H.A. Bethe, Phys. Rev. 72, 339 (1947).

[9] S.-Y. Zhu, Y. Yang, H. Chen, H. Zheng, and M. S. Zubairy, Phys. Rev. Lett., 84, 2136 (2000).

[10] A. A. Asatryan, L. C. Botten, N. A. Nicorovici, R. C. McPhedran, and C. Martijn de Sterke, Waves in Random and Complex Media, 16, 151 (2006).

[11] H. Morawits, Phys. Rev. 187, 1792 (1969).

[12] R. R. Chance, A. Prock, and R. Silbey, Phys. Rev. A, 12, 1448 (1975).

[13] A. A. Asatryan, K. Busch, R. C. McPhedran, L. C. Botten, C. M. de Sterke, and N. A. Nicorovici, Waves in Random Media, 13, 9 (2001).

[14] C. Kittel, Introduction to Solid State Physics, (John Wiley, NY, 2005). 